\title{
Stability of Pelargonidin 3-glucoside in Model Solutions in the Presence and Absence of Flavanols
}

\author{
F. Lopes-da-Silva, M.T. Escribano-Bailón and C. Santos-Buelga \\ Grupo de Investigación en Polifenoles, Unidad de Nutrición y Bromatología, \\ Facultad de Farmacia, Universidad de Salamanca, E-37007 Salamanca, Spain
}

\begin{abstract}
The stability of pelargonidin 3-glucoside ( $\mathrm{Pg} 3 \mathrm{gluc}$ ), the main anthocyanin in strawberry, has been studied in model citrate solutions $\left(\mathrm{pH} 3.5,25^{\circ} \mathrm{C}\right)$ in the absence and presence flavanols (catechin and procyanidin B3) and/or oxygen. Changes in the solutions were monitorized by HPLC-DAD/MS and UV-visible spectra. A progressive decrease in the concentration of the anthocyanin was produced according to a first-order kinetics whose rate was influenced by the composition of the medium. The availability of oxygen was the most influential factor on the stability of the anthocyanin that decreased much slower in anaerobic conditions. On the contrary, the presence of flavanols constituted a destabilizing factor for the anthocyanin. In the absence of flavanols, the anthocyanin was mostly degraded to colourless compounds, although the formation of some unknown unstable pigments with maximum absorbance in the visible region around 400-460 $\mathrm{nm}$ was also observed. In the presence of oxygen the breakdown of the heterocycle was found to constitute a major pathway for anthocyanin disappearance, whereas this mechanism was hardly produced in anaerobic conditions. In the presence of flavanols the disappearance of the anthocyanin was mostly due to condensation reactions, yielding both colourless products and xanthylium pigments. Conclusions about the structure of some of the products and pigments formed on the solutions were obtained based on their mass spectra and fragmentation patterns.
\end{abstract}

Key words: Pelargonidin, catechin, procyanidin B3, anthocyanin stability, xanthylium pigments, Strawberry

\section{INTRODUCTION}

The colour is one of the main attributes through the consumer assesses food quality and that, therefore, influences food choice and acceptability. Anthocyanins constitute a widespread family of plant pigments that are responsible for the colour of a large number fruits and fruit-derived products. These compounds are unstable with regard to $\mathrm{pH}$ and temperature variations and other factors such as light exposure, oxidation and the presence of metals and other substances, giving place to colour changes during processing and storage, which reduce the commercial value and the life span of the products containing anthocyanins. The difficulty of strawberry-derived foods (jams, juices, concentrates or purées) to maintain their attractive red colour is particularly known. In spite of many studies have been carried out, there is still an insufficient knowledge of the mechanisms subjacent to the changes of colour in strawberry foodstuffs and consequently no definitive solutions to reinforce or maintain their colour exist. Similar changes of colour are produced during ageing and storage of red wine, which are attributed to the lost of original grape anthocyanins and their substitution by complex pigments through different reactions involving anthocyanins and flavanols, without (Liao et al., 1992;

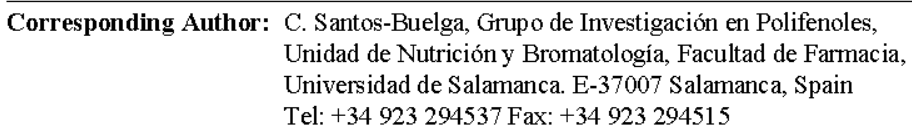


<smiles>COc1cc2c(O)cc(O)cc2[o+]c1-c1ccc(O)cc1</smiles>

(a)<smiles>Oc1cc(O)c2c(c1)O[C@H](c1ccc(O)c(O)c1)[C@H](O)C2</smiles>

(b)<smiles>OC1=CC([C@H]2Oc3cc(O)cc(O)c3[C@H](Cc3c(O)cc(O)c4c3O[C@H](c3ccc(O)c(O)c3)[C@H](O)C4)[C@H]2O)=CCC1O</smiles>

(c)

Fig. 1: Chemical structures of Pg3gluc (a), (+)-catechin (b) and procyanidin dimer B3 (c)

Somers, 1971; Boulton, 2001; Kunsági-Máté et al., 2006; Hermosín-Gutiérrez et al., 2005) or with the involvement of other compounds, such as acetaldehyde (Bakker et al., 1993; Francia-Aricha et al., 1997; Rivas-Gonzalo et al., 1995; Timberlake and Bridle, 1976), pyruvic acid (Bakker et al., 1997; Bakker and Timberlake, 1997; Fulcrand et al., 1998; Romero and Bakker, 1999), vinylphenols (Cameira dos Santos et al., 1996; Fulcrand et al., 1996; Hayasaka and Asenstorfer, 2002), a-ketoglutaric acid (Benabdeljalil et al., 2000), acetone (Hayasaka and Asenstorfer, 2002), or glyoxylic acid (Es-Safi et al., 1999; Fulcrand et al., 1997). As a result of these reactions, new flavylium-derived compounds, pyranoanthocyanins and xanthylium pigments with different chromatic features are produced. In addition to anthocyanins, the strawberry also contains flavanols and, therefore, it can be supposed that some of the previous processes may also occur in their derived products and contribute to their changes of colour. The anthocyanin composition in the strawberry is characterised by the presence of pelargonidin 3-glucoside (Pg3gluc, Fig. 1) as major pigment, constituting 77-95\% of total anthocyanins (Goiffon et al., 1999; Lopes da Silva et al., 2007). Strawberry flavanols are mainly based on catechin (de Pascual-Teresa et al., 2000b; Thompson et al., 1972), being (+)-catechin the majority compound followed by the procyanidin dimer B3 (catechin-4,8-catechin, Fig. 1); these two compounds represent around 40 and $13 \%$ of the total content of flavanols of low degree of polymerisation in the strawberry, respectively (Lopes da Silva et al., 2002). The aim of the present work was to study the behaviour of Pg3gluc in model assays in the absence and presence of oxygen and flavanols (catechin and procyanidin B3), so as to conclude about the mechanisms involved in their instability.

\section{MATERIALS AND METHODS}

\section{Standards}

$(+)$-Catechin, ( $( \pm)$-taxifolin, $p$-hydroxybenzoic acid and 2,4,6-trihydroxybenzaldehyde were purchased from Sigma Chemical Co. (St. Louis, MO). 


\section{Isolation of Pelargonidin 3-Glucoside}

Strawberries of the Camarosa variety were homogenized in methanol containing $0.1 \% \mathrm{HCl}$, maintained at $-30^{\circ} \mathrm{C}$ overnight and further centrifuged $\left(4000 \mathrm{~g}, 15 \mathrm{~min}, 2^{\circ} \mathrm{C}\right)$. Water was added to the supernatant and the methanol removed in a rotary evaporator at $30^{\circ} \mathrm{C}$. The aqueous extract obtained was purified by liquid chromatography in an open column filled with a mixed stationary phase composed of $20 \%$ Polyclar AT and $80 \%$ silica gel G60 to remove sugars and acids, as described by Escribano-Bailón et al. (2002) Isolation of Pg3gluc was further carried out by semipreparative-HPLC. An RP Nova-Pak C18 cartridge ( $6 \mu \mathrm{m}, 60 \AA, 25 \times 100 \mathrm{~mm}$ ) (Waters, Milford, MA) was used. Solvents were (A) 5\% acetic acid and (B) 100\% HPLC grade methanol. The elution profile was as follows: $0 \mathrm{~min}, 90 \% \mathrm{~A}, 10 \% \mathrm{~B} ; 5-20 \mathrm{~min}, 85 \% \mathrm{~A}, 15 \% \mathrm{~B} ; 20-25 \mathrm{~min}, 85 \% \mathrm{~A}, 15 \% \mathrm{~B} ; 25-30 \mathrm{~min}, 82 \% \mathrm{~A}$, $18 \% \mathrm{~B} ; 30-50 \mathrm{~min}, 65 \% \mathrm{~A}, 35 \% \mathrm{~B}$. The flow rate was $10 \mathrm{~mL} \mathrm{~min} \mathrm{~m}^{-1}$. Detection was carried out at $280 \mathrm{~nm}$ and the peaks were collected in a fraction collector. Fractions containing the anthocyanin were mixed, the solvents eliminated under vacuum and the pigment transferred to water and freeze-dried. The purity of the anthocyanin was checked by HPLC-DAD/MS.

\section{Preparation of Procyanidin B3}

It was synthesized as described by de Freitas (1995). The method is based on the reduction of $( \pm)$-taxifolin by sodium borohydride followed by the condensation between the carbonium ion $\left(\mathrm{C}^{+}\right)$, formed in acidic media and $(+)$-catechin. Procyanidin B3 was isolated from the reaction mixture by fractionation on a Sephadex LH-20 column and further purified by semi-preparative HPLC as described by de Pascual-Teresa et al. (1998). The compound was freeze-dried and its purity checked by HPLC-DAD/MS.

\section{Model Solutions}

Five solutions were prepared in citrate buffer $0.1 \mathrm{M}$ at pH 3.5. Solutions $\mathrm{A}$ and B contained $2 \mathrm{mM}$ Pg3gluc; solutions C and D contained a mixture of Pg3gluc ( $2 \mathrm{mM})$, catechin (1.5 mM) and procyanidin B3 $(1.0 \mathrm{mM})$ and solution E contained Pg3gluc $(2 \mathrm{mM})$ and procyanidin B3 $(1.5 \mathrm{mM})$. All the solutions were kept in a glass vial closed with a septum and stored in dark at $25^{\circ} \mathrm{C}$. Solutions B and D were purged with $\mathrm{N} 2$ during $2 \mathrm{~min}$ before sealing the vial. Oxygen exposure was not avoided when vials of solutions A, C and E were closed. Samples were taken periodically over 3 months by puncturing the septum and analysed by HPLC-DAD/MS. Solution B was followed during 6 months. The study was conducted in 2004 at the laboratory of Food Science, University of Salamanca (Spain).

\section{HPLC-DAD/MS Analy sis}

Analyses were carried out using a Hewlett-Packard 1100 chromatograph with a quaternary pump and a Diode Array Detector (DAD) coupled to an HP Chem Station. An Aqua C18, $5 \mu \mathrm{m}$ $\left(4.6 \times 150 \mathrm{~mm}\right.$ ) (Phenomenex, Torrance, CA) column was used thermostated at $30^{\circ} \mathrm{C}$. The mobile phase was (A) $0.1 \%$ trifluoroacetic acid in water and (B) HPLC-grade acetonitrile. The elution gradient established was $0 \mathrm{~min}, 90 \% \mathrm{~A}, 10 \% \mathrm{~B} ; 5-20 \mathrm{~min}, 85 \% \mathrm{~A}, 15 \% \mathrm{~B} ; 20-25 \mathrm{~min}, 85 \% \mathrm{~A}, 15 \% \mathrm{~B}$; $25-30 \mathrm{~min}, 82 \% \mathrm{~A}, 18 \% \mathrm{~B} ; 30-50 \mathrm{~min}, 65 \% \mathrm{~A}, 35 \% \mathrm{~B}$. The flow rate was $0.5 \mathrm{~mL} \mathrm{~min}^{-1}$. Detection was carried out at $280,330,440$ and $500 \mathrm{~nm}$. MS detection was also performed using a Finnigan LCQ detector (San Jose, CA) equipped with an ESI source and anion trap mass analyser, which were controlled by the LCQ Xcalibur software. The mass spectrometer was connected to the HPLC system via the DAS cell outlet. Nitrogen was used as both auxiliary and shealth gase at flow rates of 6 and $1.2 \mathrm{~L} \mathrm{~min}^{-1}$, respectively. The capillary voltage was $4 \mathrm{~V}$ and the capillary temperature $195^{\circ} \mathrm{C}$. Spectra were recorded in positive ion mode between $\mathrm{m} / \mathrm{z} 150$ and 1500 . The MS detector was programmed to perform a series of three consecutive scans: a full mass scan, a zoom scan of the most abundant ion in the first scan and an MS-MS scan of the most abundant ion, using a normalized collision energy of $45 \%$ 


\section{RESULTS AND DISCUSSION}

\section{Kinetics of Pg3gluc Disappearance}

The changes in the composition of the solutions were followed by analysis of HPLC-DAD/MS. In all cases a progressive decrease in the content of Pg3gluc was produced, although at a different rate depending on the solution. The disappearance of the anthocyanin took place according to an apparent first-order kinetics $\left(\mathrm{C}=\mathrm{C} 0 . \mathrm{e}^{-\mathrm{kt}}\right.$ ), as previously observed for the degradation of Pg3gluc in juices (Garzon and Wrolstad, 2001) and concentrates of strawberry (Garzon and Wrolstad, 2002; Özkan et al., 2002), as well as in model solutions of different anthocyanins (Baublis et al., 1994; Cemeroglu et al., 1994; Özkan, 2002; Giusti and Wrolstad, 1996). The Table 1 shows the reaction rate constants $(\mathrm{k})$ and the half-life time $\left(\mathrm{t}_{1 / 2}\right)$ of Pg3gluc in the different solutions determined using linear regression from plot of $\ln [\mathrm{C}]$ vs time. For this calculation only the two first months of storage were considered, since, except for the solution B, small or no amounts of anthocyanin were detected after that time. The rates of disappearance were of the same order of magnitude than the ones observed by Dueñas et al. (2006) for the disappearance of malvidin 3-glucoside (Mv3gluc) in the presence of equimolar concentrations of epicatechin in model solutions prepared in $20 \mathrm{mM}$ ammonium acetate at the same $\mathrm{pH}$ value $\left(\mathrm{k}=4.92 \times 10^{-2}\right.$ days $^{-1}, \mathrm{t}_{1 / 2}=14.1$ days $)$.

As it could be expected, the disappearance of the anthocyanin was much slower when oxygen was avoided. The faster degradation in the presence of oxygen was already reported either for pure anthocyanins (Poei-Langston and Wrolstad, 1981; Kallio et al., 2000) or food matrixes (Clydesdale et al., 1978). The adjustment to a first-order kinetics was worse in the solutions containing catechin and/or procyanidin B3, suggesting that other processes may be contributing to the disappearance of Pg3gluc. Indeed, the presence of flavanols in the medium constitutes a destabilizing factor for the anthocyanin. In the conditions of the assay, the effect of the flavanols even counteracts the protection offered by the anaerobic storage, reducing the half-life of the anthocyanin to values closed to the one observed in the solutions prepared without avoiding oxygen. Poei-Langston and Wrolstad (1981) also found a destabilizing effect of the flavanols in model solutions containing Pg3gluc and catechin in a molar ratio of 1:6.7, greater than the one used in our solutions. In the assays carried out by those authors, the decrease of the anthocyanin was even faster in the mixtures Pg $3 g l u c:$ catechin maintained in the absence of oxygen than in the solutions containing only anthocyanin in the presence of oxygen.

The rate of disappearance of the flavanols in the solution is slower than that of the anthocyanin (Table 1) and did not fit well a first-order kinetics, as also observed by Dueñas et al. (2006). The time of half-life was greater for catechin than for procyanidin B3, which may be explained by the lability of the interflavanic linkage and easier oxidation in the case of the dimer (Oszmianski et al., 1985; de Freitas et al., 1998; Francia-Aricha et al., 1998). Furthermore, the interflavanic cleavage of the procyanidin releases free catechin, thus contributing to moderate the decrease of this latter.

\section{Changes in the Solutions Containing Only Pg3gluc}

In the solutions containing only Pg3gluc, the disappearance of the anthocyanin is accompanied by the formation of new compounds (Fig. 2). The Table 2 shows the characteristics (wavelength of

Table 1: Rate of disappearance of pelargonidin 3-glucoside (Pg3gluc), catechin (c) and procy anidin B3 in the different model solutions assayed

\begin{tabular}{|c|c|c|c|c|c|c|}
\hline \multirow[b]{2}{*}{ Solution } & \multirow[b]{2}{*}{ Composition } & \multicolumn{3}{|l|}{ Pg3gluc } & \multirow{2}{*}{$\begin{array}{l}\text { Catechin } \\
t_{1 / 2} \text { (days) }\end{array}$} & \multirow{2}{*}{$\begin{array}{l}\text { Procyanidin B3 } \\
t_{1 / 2} \text { (days) }\end{array}$} \\
\hline & & $\mathrm{k}\left(\right.$ days $\left.^{-1}\right)$ & $t_{12}$ (days) & $\mathrm{R}^{2}$ & & \\
\hline A & Pg3gluc & $5.07 \times 10^{-2}$ & 13.6 & 0.9788 & & \\
\hline B & Pg3gluc (anaerobic) & $1.44 \times 10^{-2}$ & 48.0 & 0.9817 & & \\
\hline $\mathrm{C}$ & Pg3gluc $+\mathrm{C}+\mathrm{B} 3$ & $5.52 \times 10^{-2}$ & 12.5 & 0.9079 & 17.8 & 12.3 \\
\hline $\mathrm{D}$ & Pg3gluc $+\mathrm{C}+\mathrm{B} 3$ (anaerobic) & $4.28 \times 10^{-2}$ & 16.9 & 0.8249 & 35.9 & 29.6 \\
\hline $\mathrm{E}$ & Pg Pg3gluc 3g + B3 & $4.88 \times 10^{-2}$ & 14.2 & 0.8929 & & 16.7 \\
\hline
\end{tabular}




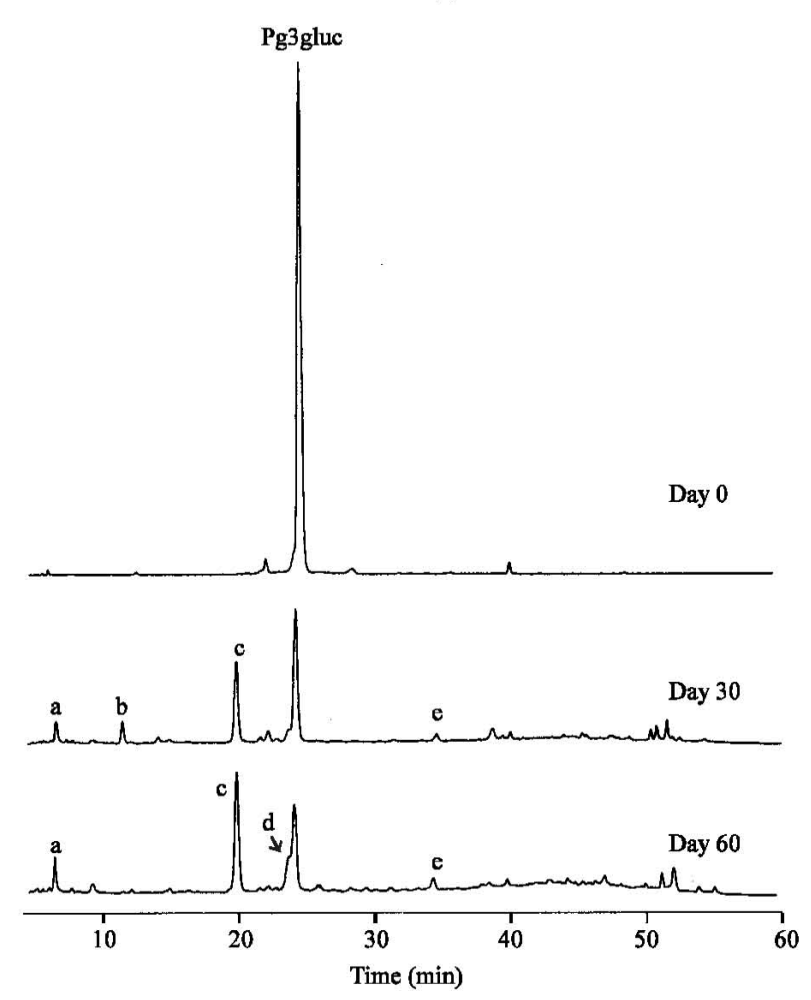

Fig. 2: HPLC chromatogram recorded at $280 \mathrm{~nm}$ of solution $\mathrm{A}$ at days 0,30 and 60 of assay. The scale (mAbs units) is the same in all cases

Table 2: Absorption and mass spectral characteristics of the products found in solutions A and B

\begin{tabular}{lccll}
\hline Peak & $\lambda \max (\mathrm{nm})$ & Molecularion $(\mathrm{m} / \mathrm{z})$ & $\mathrm{MS}^{2}$ fragment ions $(\mathrm{m} / \mathrm{z})^{*}$ & Peak identity \\
\hline a & 290 & 487 & $457,325,317$ & unknown \\
b & 254 & & & p-hydroxybenzoic acid \\
c & 291 & 155 & 137 & $2,4,6$-trihydroxybenzaldehyde \\
d & 292 & 257 & & unknown \\
e & 340 & 865 & 703,541 & Anthocyanin dimer \\
\hline
\end{tabular}

*Fragment ions are indicated in decreasing order of relative abundance

maximum absorption, molecular ion and $\mathrm{MS}^{2}$ fragments) of peaks a-e for which information could be obtained.

Compound a showed a molecular ion at $\mathrm{m} / \mathrm{z} 487$, to which no suitable structure could be fitted. One of its $\mathrm{MS}^{2}$ fragments corresponds to the loss of glucose ( $\mathrm{m} / \mathrm{z}$ at $\left.325,-162 \mathrm{amu}\right)$, indicating that the compound directly derives from the anthocyanin. Its higher mass in relation to $\mathrm{Pg} 3 \mathrm{gluc}$ suggests that it could be formed by the reaction of the anthocyanin with a compound of low molecular weight, which may derive fro the citric acid used in the preparation of the solutions or a product from the degradation of the anthocyanin.

Peaks $b$ and $c$ were observed in solution $A$ and identified as p-hydroxybenzoic acid (p-HB) and 2,4,6-trihydroxybenzaldehyde (THB), respectively, by comparison of their UV spectra and retention time with standards. The identity of THB could also be confirmed by its mass spectrum (molecular ion at $\mathrm{m} / \mathrm{z} 155$ ), whereas no ionisation of $\mathrm{p}-\mathrm{HB}$ was produced in the MS conditions used. These compounds would derive from the fragments corresponding to the anthocyanin rings A (THB) and B (p-HB) released after breakdown of the heterocycle, according to the mechanism proposed by Furtado et al. (1993) and Piffaut et al. (1994). The presence of the aglycone (Pelargonidin, Pg) was 
irregularly detected in the chromatograms during the first of month of assay (Fig. 3), suggesting that the separation of the glucose constitutes a previous step in the anthocyanin degradation. The levels of the aglycone detected were always low in agreement with its assumed instability. THB and p-HB were not stable in the solution and progressed to the formation of other unidentified structures. The amounts of $\mathrm{p}-\mathrm{HB}$ accumulated were lower and its disappearance faster than those of THB, indicating a greater reactivity. In the absence of oxygen (solution B) hardly formation of THB and p- HB was detected, denoting that the anthocyanin breakdown does not represent a relevant mechanism for anthocyanin disappearance in anaerobic conditions.

Another major product found in the solutions was peak $d$ whose formation was observed both in the presence and absence of oxygen. In fact, this was the only important peak detected in the chromatograms of solution B maintained in anaerobic conditions (Fig. 4). This compound showed a molecular ion at $\mathrm{m} / \mathrm{z} 257$ that produced no $\mathrm{MS}^{2}$ fragments in the conditions employed. No suitable structure could be assigned to this ion. The compound e mostly accumulated in the solutions when low levels of Pg3gluc existed, suggesting that it does not derive directly from the anthocyanin but an intermediate product should exist that could not be detected.

Peak e showed a molecular ion at $\mathrm{m} / \mathrm{z} 865$ twice the mass of Pg3gluc and in its fragmentation the successive loss of two hexose residues is produced (fragments at $\mathrm{m} / \mathrm{z} 703$ and $\mathrm{m} / \mathrm{z} \mathrm{541}$ ). These characteristics are coherent with a dimer constituted by two anthocyanin moieties. In assays carried out with Mv3gluc other authors (Salas et al., 2005; Vidal et al., 2004) had already observed the formation of anthocyanin dimers, for which flaven-flavylium C-C linked or flavan-flavylium doubly linked structures were proposed. The compound e did not show an absorption maximum in the region of $500 \mathrm{~nm}$ pointing out that it did not contain flavylium units. Thus, two possible structures, i.e., flaven-flaven (Fig. 5a) and doubly-linked flavan-flaven (Fig. 5b) can be suggested as possible.

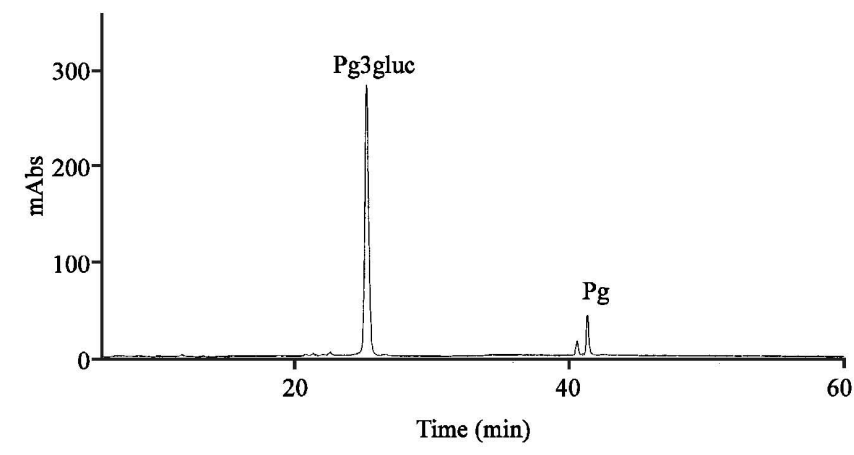

Fig. 3: HPLC chromatogram recorded at $520 \mathrm{~nm}$ of solution A at day 30 of assay where the presence of pelargonidin $(\mathrm{Pg})$ can be seen

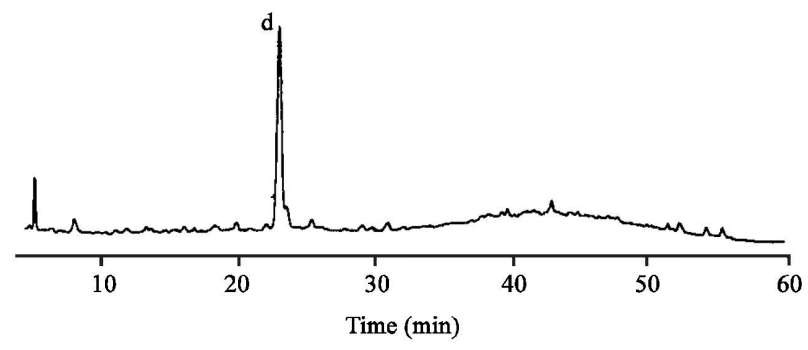

Fig. 4: HPLC chromatogram recorded at $280 \mathrm{~nm}$ of solution B at day 180 of assay 

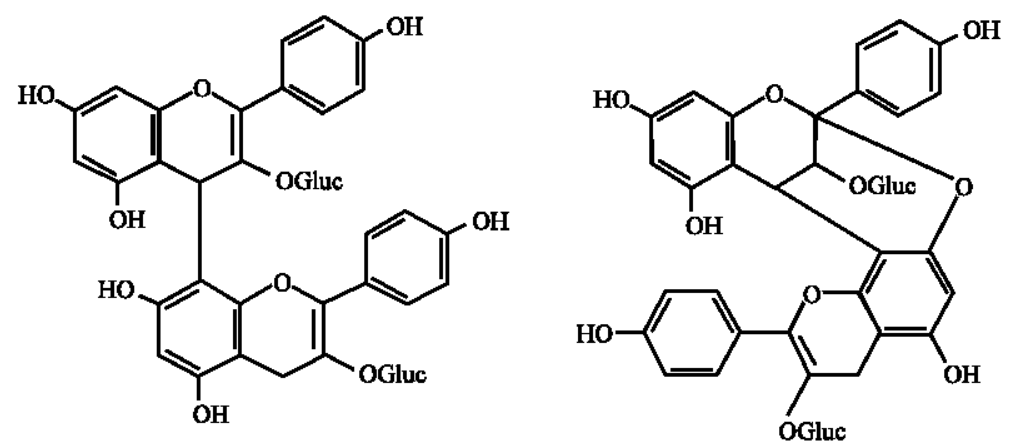

Fig. 5: Suggested structures for compound e ( $\mathrm{m} / \mathrm{z}$ at 865$)$
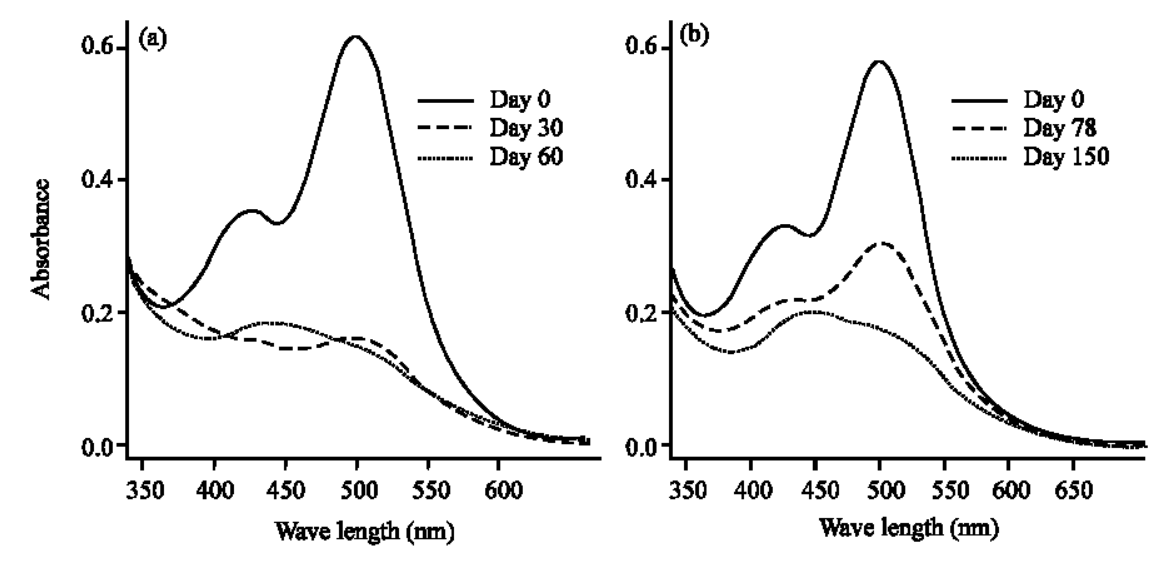

Fig. 6: Visible absorption spectra in solutions A (a) and B (b) recorded at different days of the assay

During storage, a progressive change in the colour of the solution from bright red to red orange and yellow orange was produced (Fig. 6). The formation of turbidity was also observed in the second month of assay and further accumulation of a brown sediment. The compounds detected in the chromatograms above discussed were mostly colourless and, therefore, other substances must have been contributing to the colour when low or no levels of the anthocyanin exist in the solution. As it can be seen in Fig. 2, in addition to peaks a-e the appearance of other peaks and a hump were also observed in the end part of the chromatograms. Those peaks, some of which showed absorption in the region of 400-460 $\mathrm{nm}$, were not reproducible in the successive analyses, indicating that they corresponded to transient substances, quite possibly evolving to the formation of polymers responsible for the brown precipitate. No reliable information about those compounds could be obtained by mass spectrometry and, therefore, their nature remains unknown. The greater formation of these compounds was observed when hardly Pg3gluc existed in the medium, suggesting that they rather derived from products of the degradation of the anthocyanin and/or the citric acid used in the preparation of the model solutions. The evolution of the colour in solution B was much slower than in solution $\mathrm{A}$, in accordance with the increased stability of the anthocyanin due to the restriction of oxygen. The red colour was maintained during a substantial part of the storage and only in the fourth month, when the levels of Pg3gluc were already low, a change to orange started to be appreciated. The formation of a light precipitate was also observed at the end of the six months of assay. In this solution, the products responsible for the colour when the anthocyanin decreased could not be detected in the chromatograms. 


\section{Changes in the Solutions Containing Pg3gluc and Flavanols}

In solutions C-E different pattern of changes was observed with regard to A and $\mathrm{B}$. The formation of four colourless compounds (P1, P2, P5 and P6) and four pigments (P3, P4, P7 and P8) was detected (Fig. 7) about which information (UV-visible and mass spectra) could be obtained (Table 3). The same compounds were observed in the presence and absence of oxygen, although in the latter case the composition changes took place more slowly.

Peak P5 corresponded to the unknown product $d$ found in solutions A and B. This was the only important compound derived from the degradation of Pg3gluc detected in solutions C-E, where no significant formation of THB and p-HB was observed, suggesting that the heterocycle breakdown is not a preferential pathway for the anthocyanin loss in the presence of flavanols. The remaining compounds detected in solutions C-E derived from different processes of condensation between the anthocyanin and the flavanols, which constituted the most important mechanisms for the anthocyanin disappearance in these solutions, either in the presence and absence of oxygen.

The compounds P1 and P2 had the same molecular ion ( $\mathrm{m} / \mathrm{z}$ at 577$)$ and similar fragmentation pattern. Their presence in the solution $\mathrm{E}$ that contained the dimer B3 as the only flavanol confirmed the implication of this procyanidin in their formation. The molecular ion would fit A-type

\begin{tabular}{lllll}
\multicolumn{5}{l}{ Table 3: Absorption and mass spectral characteristics of the products found in solutions C-E } \\
\hline Peak & $\lambda \max (\mathrm{nm})$ & Molecular ion $(\mathrm{m} / \mathrm{z})$ & $\mathrm{MS}^{2}$ fragment ions $(\mathrm{m} / \mathrm{z})^{*}$ & Peak identity \\
\hline P1 & 281 & 577 & $425,407,299,409,559$ & Flavanol-anthocyanidin condensed dimer \\
P2 & 274 & 577 & $425,407,299,409$ & Flavanol-anthocyanidin condensed dimer \\
P3 & 276,447 & 741 & $527,375,363,405$ & Xanthylium pigment \\
P4 & 273,436 & 453 & $301,435,257,283$ & Xanthylium pigment \\
P5 & 292 & 257 & & Unknown (peak d) \\
P6 & 275 & 723 & 571,561 & Anthocyanin-flavanol condensed dimer \\
P7 & 271,434 & 861 & $723,741,553,571,419$ & Xanthylium pigment \\
P8 & 267,425 & 573 & $435,453,283$ & Xanthylium pigment
\end{tabular}

*Fragment ions are indicated in decreasing order of relative abundance
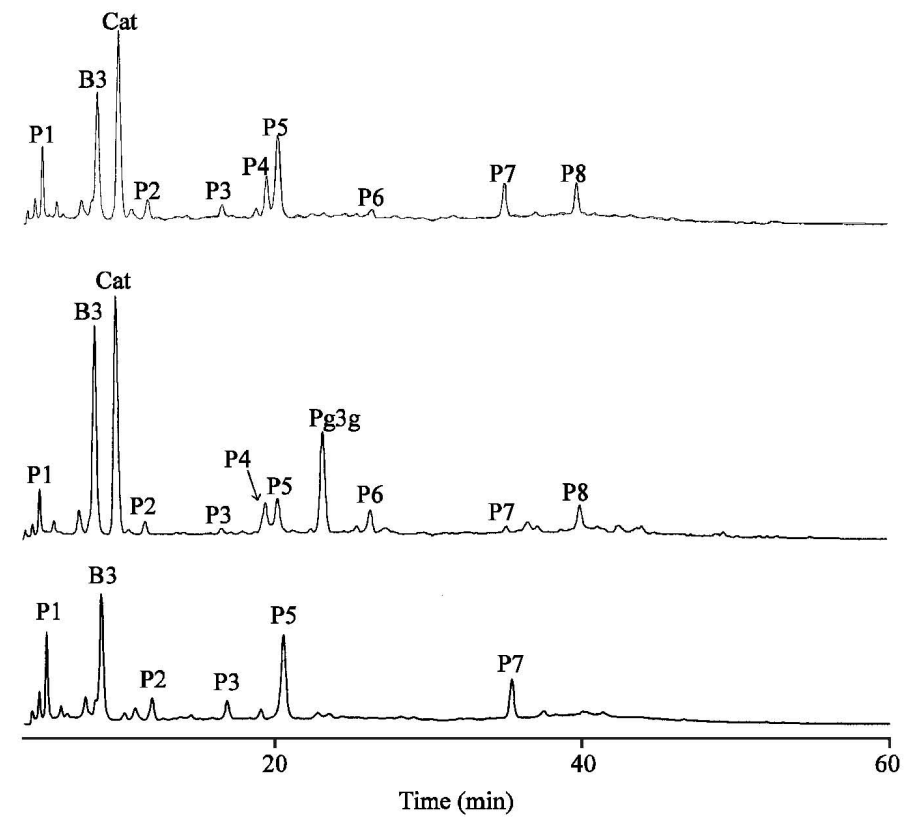

Fig. 7: HPLC chromatograms recorded at $280 \mathrm{~nm}$ of solution C (upper chromatogram), solution D (middle) and solution E (bottom) after 80 days of storage. The scale (mAbs units) is the same in all cases 


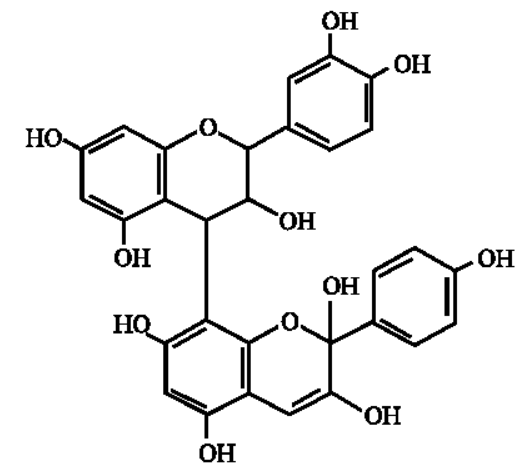

Fig. 8: Suggested structure for peak P1 ( $\mathrm{m} / \mathrm{z}$ at 577). P2 would have similar structure but C4-C6 linked

proanthocyanidin dimers, which might derive from the structural rearrangement of B3 following breakage of its interflavanic bond. However, this possibility was discarded, since such type of dimers should elute later than procyanidin B3 in the chromatograms using reversed-phase columns (Santos-Buelga et al., 2003). Another possibility is that they result from the direct condensation of the carbocation $\mathrm{C}^{+}$, released after the interflavanic breakdown of procyanidin $\mathrm{B} 3$, over the nucleophilic positions $\mathrm{C} 8$ and $\mathrm{C} 6$ of the carbinol form of Pg3gluc to yield F-A dimers where the anthocyanin moiety is a flavene hydrated form (Fig. 8). The $\mathrm{MS}^{2}$ fragmentation the compounds would be coherent with such a type of structure. The main fragment (m/z at 425, -152 amu) would correspond from the Retro Diels-Alder decomposition (RDA) of catechin, which has been seen to take place preferentially on the upper unit (de Pascual-Teresa et al., 2000a; Friedrich et al., 2000). Further fragmentation of this ion would give rise to the fragments at $\mathrm{m} / \mathrm{z} 407$ (loss of water) and m/z 299 (-126 amu, loss of phloroglucinol after heterocyclic ring fission of the catechin unit). Minor signals at $\mathrm{m} / \mathrm{z} 559$ and $\mathrm{m} / \mathrm{z} 409$ are interpreted as the loss of water from the hydroxyl group at $\mathrm{C} 3$ of the flavanol moiety ( $-18 \mathrm{amu}$ ) and catechin RDA together with the loss of the hydroxyl group at $\mathrm{C} 2$ of the anthocyanin moiety (-152-16 amu), respectively.

The structure proposed in Fig. 8 for P1 and P2 lacks the glucose attached to the anthocyanidin moiety. The glucosylated precursor of such a structure could be expected to be stable, however, it was not detected in any of the HPLC-DAD/MS analyses made. Thus, if the proposed structure is confirmed, it might have formed from the direct reaction of the flavanol carbocation with the anthocyanin aglycone $(\mathrm{Pg})$. Taking into account that the production of the aglycone constitutes a first step in the anthocyanin breakdown (Piffaut et al., 1993), its possible reaction with the flavanol might explain the low rate of products from Pg degradation (THB and p-HB) observed in the solutions containing flavanols.

Compound P6 showed a molecular ion at $\mathrm{m} / \mathrm{z} 723$ with $\mathrm{MS}^{2}$ fragments at $\mathrm{m} / \mathrm{z} 571$ and $\mathrm{m} / \mathrm{z} 561$ corresponding to the RDA fission of catechin and the loss of a glucose moiety respectively. These characteristics fit a dimer containing catechin and Pg3gluc residues. This compound was not detected in the solution E (Pg3gluc+ procyanidin B3), pointing out that the monomeric catechin was the flavanol involved in is formation. The possibility that P6 was a catechin-Pg3gluc dimer C6'-C8 linked was discarded, since no RDA fission of catechin could have been produced. On the other hand, the formation of such a type of dimers would require the previous oxidation of catechin to an $o$-quinone (Sarni-Manchado et al., 1997) that would not be expected to occur in the solution D (prepared avoiding oxygen) in which P6 is a relevant product. This observation linked to the fact that flavanol monomers are unable to produce a $\mathrm{C4}^{+}$carbocation, strongly suggested that catechin was located in the lower unit of the condensed dimer. Thus, two possible structures that fit the molecular ion found for P6 can be 


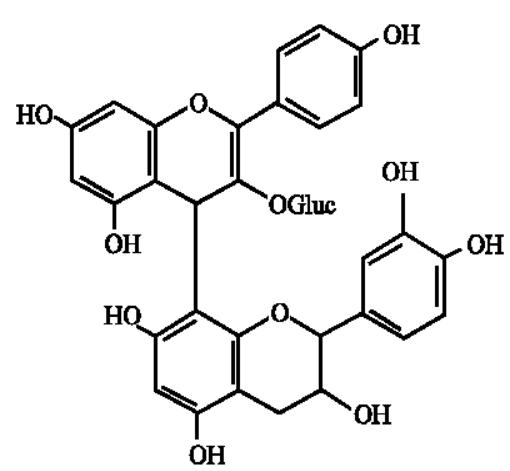

(a)

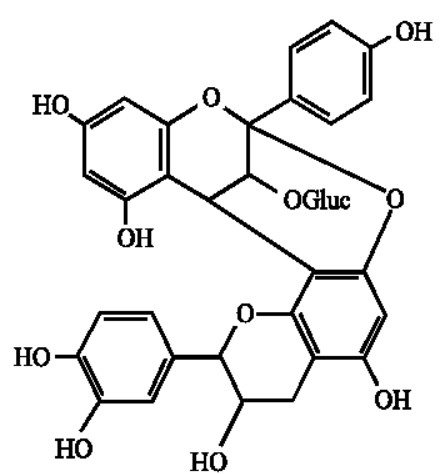

(b)

Fig. 9: Suggested structures for product P6 (m/z at 723$)$

proposed corresponding to A-F dimers in which anthocyanin and catechin would be either C-C linked (Fig. 9a) or doubly-linked like in A-type proanthocyanidins (Fig. 9b).

In the fragmentation of anthocyanin-flavanol singly-linked species in positive ion mode, the preferential loss of the glucose moiety and further catechin RDA of the released aglycone would be expected (Gonzalez-Paramas et al., 2006; Salas et al., 2003). However, the production of a fragment from the direct catechin RDA that could even predominate over the fragment corresponding to the loss of glucose was observed for A type dimers (Dueñas et al., 2006; Remy-Tanneau et al., 2003). In our case, the main fragment produced in the $\mathrm{MS}^{2}$ fragmentation of $\mathrm{P} 6$ corresponded to the direct catechin RDA fission ( $\mathrm{m} / \mathrm{z}$ at 571), whereas a minor signal was observed for the fragment derived from the loss of the glucose ( $\mathrm{m} / \mathrm{z}$ at $561,10 \%$ of relative abundance), which might support an A-type nature for P6 (Fig. 9b).

A flaven-flavan dimer like the one in Fig. 9a would be expected to be the first species formed after the condensation anthocyanin-catechin. Further rearrangement of this adduct could be produced to either a coloured xanthylium structure (Jurd, 1969; Liao et al., 1992; Somers, 1971) or a colourless A-type derivative (Jurd, 1969; Jurd and Waiss, 1965). The preferential formation of this latter in anaerobic media was found by Bishop and Nagel (1984) and Remy-Tanneau et al. (2003). In our assays P6 accumulated in higher amounts in solution D that had been purged with N2 than in solution $\mathrm{C}$ where oxygen was not avoided and only traces of P6 were detected. This set of observations would contribute further support to the A-type nature of this compound. On the other hand, greater formation of xanthylium pigments was observed in the solution $\mathrm{C}$, suggesting that in oxidative conditions a rearrangement of the A-F adducts towards xanthylium derivatives is produced.

The products P4 (m/z at 453) and P8 (m/z at 573) showed absorption spectra corresponding to xanthylium structures (Fig. 10). In previous studies of our group the formation of a compound with the same molecular ion and similar UV-visible spectrum as P4 was found in wine model assays containing catechin and Mv3gluc (Santos-Buelga et al., 1999). That pigment was suggested to derive from the glyoxylic acid-mediated condensation between catechin and phloroglucinol moieties, in accordance with the mechanism described by Fulcrand et al. (1997). In that case, the glyoxylic acid would result from the oxidation of the tartaric acid used in the preparation of the model solutions. In the current assays the presence of glyoxylic acid in the solutions would not be expected and suggest that other mechanisms are involved in the formation of P4.

Xanthylium pigments can also be formed from the direct condensation between flavylium cations and structures containing phloroglucinol nuclei (like flavanols) as early described by Jurd (1969). Such a mechanism was also used by Liao et al. (1992) and Santos-Buelga et al. (1995) to explain the 


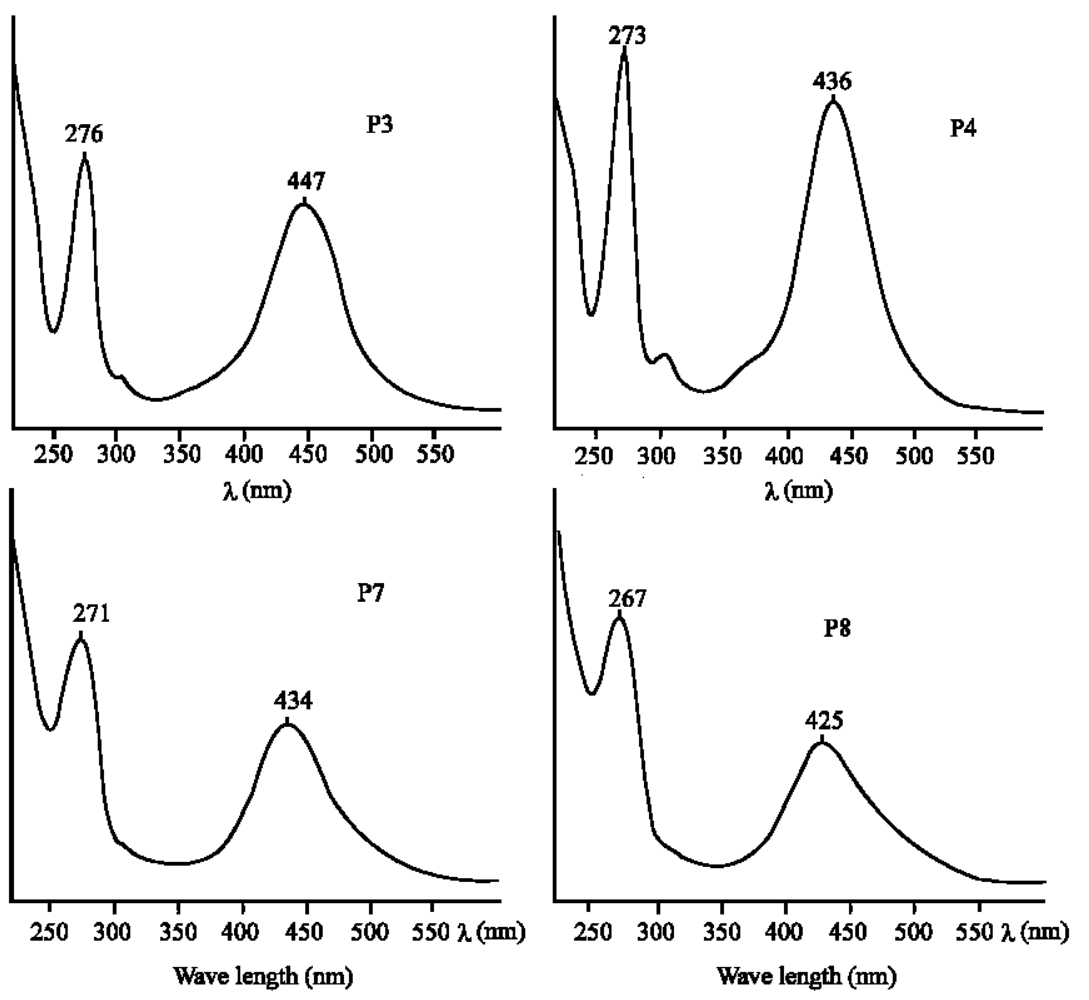

Fig. 10: UV-visible spectra of new xanthylium pigments formed in solutions C-E

formation of yellow products in wine model solutions. Quite recently Dueñas et al. (2006), in model solutions of Mv3gluc and epicatechin in ammonium formate, found the formation of a xanthylium derivative with same molecular ion ( $\mathrm{m} / \mathrm{z}$ at 453$)$ and similar UV-visible spectrum as P4 and also suggested a mechanism based on the direct condensation anthocyanin-flavanol to explain its formation. The mechanism proposed by these authors would also involve the $\mathrm{C} 2-\mathrm{C} 3$ oxidative cleavage of the anthocyanin heterocycle to yield an intermediate malvone, as previously described by Hrazdina and Franzese (1974). The process would require an acidic $\mathrm{pH}$ since under neutral or alkaline conditions the separation of the anthocyanin Bring with formation of a coumarin would be expected (Hrazdina and Franzese, 1974). Based on the proposal of Dueñas et al. (2006), a possible pathway for the formation of pigments P4 and P8 in our solutions is suggested in Fig. 11.

The structures proposed for pigments P4 and P8 in Fig. 11 fit their $\mathrm{MS}^{2}$ fragmentation patterns. Regarding P4 (m/z at 453) the main fragment at m/z 301 is attributed to catechin RDA fission [M+H$152]^{+}$; the signal at $\mathrm{m} / \mathrm{z} 257[\mathrm{M}+\mathrm{H}-152-44]^{+}$would correspond to the additional loss of the carboxyl group. The fragments at $\mathrm{m} / \mathrm{z} 435$ and $\mathrm{m} / \mathrm{z} 283$ would derive from the loss of water $[\mathrm{M}+\mathrm{H}-18]^{+}$ and further RDA [M+H-18- 152] $]^{+}$, respectively. In the case of $\mathrm{P} 8(\mathrm{~m} / \mathrm{z}$ at 573$)$ the main fragment $(\mathrm{m} / \mathrm{z}$ at 435$)$ would result of the loss of the p-hydroxybenzoyl moiety $(-138 \mathrm{amu})$ corresponding to anthocyanin ring- $\mathrm{B}$ and the ion at $\mathrm{m} / \mathrm{z}$ at 453 from the alternative breakdown of the same ring $(-120 \mathrm{amu})$. Further RDA of the ion at $\mathrm{m} / \mathrm{z} 435$ would yield the fragment at $\mathrm{m} / \mathrm{z} 283$. Pigments $\mathrm{P} 3(\mathrm{~m} / \mathrm{z}$ at 741$)$ and $\mathrm{P} 7(\mathrm{~m} / \mathrm{z}$ at 861$)$ would be similar to $\mathrm{P} 4$ and $\mathrm{P} 8$, but derived from procyanidin B3 instead of catechin.

The formation of xanthylium pigments according to the mechanism of Fig. 11 involves oxidative steps, which makes it difficult to justify their presence in the solution D prepared in anaerobic 


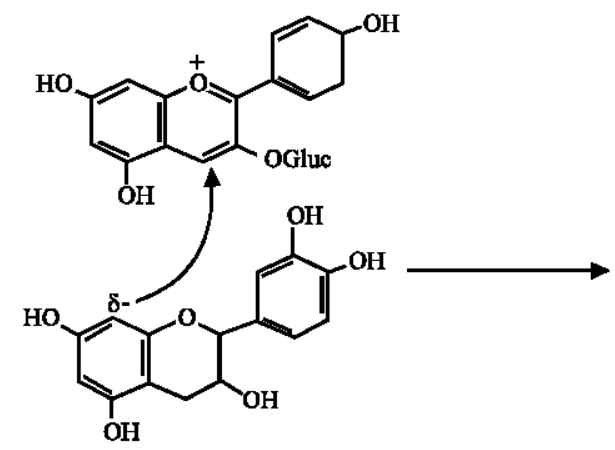<smiles></smiles><smiles>COC(O)=C(c1c(O)cc(O)cc1O[PH](=O)c1ccc(O)cc1)c1c(O)cc(O)c2c1OC(C1=CCC(O)C(O)=C1)C(O)C2</smiles><smiles>COC1=C(c2c(O)cc(O)c3c2OC(c2ccc(O)c(O)c2)C(O)C3)c2c(O)cc(O)cc2OC1(O)c1ccc(O)cc1</smiles><smiles>[3H]C</smiles><smiles></smiles>

P8<smiles></smiles>

P4

Fig. 11: Proposed pathway for the formation of compounds P4 and P8

conditions. Obviously the incorporation of small amounts of oxygen to the vials during sampling might be an explanation. On the other hand, Somers and Evans (1986) observed that similar pattern of changes were produced in the colour composition of red wines conserved in the absence and presence of oxygen and Jurd (1967) had also showed that the flavylium cation could act as an oxidant in the process of formation of new pigments derived from the condensation between flavylium salts (like the anthocyanins) and catechin. These observations might support the formation of yellow pigments in the solution $\mathrm{D}$ even if oxygen was not available.

In the solutions containing anthocyanins and flavanols a change of colour was produced from bright red to brown-orange. Furthermore, the accumulation of a light brown precipitate was also observed at the end of the assay. In the visible spectra of the solutions a progressive decrease in the absorbance at $500 \mathrm{~nm}$ and an increase in the region of $430 \mathrm{~nm}$ was produced (Fig. 12), attributable to 

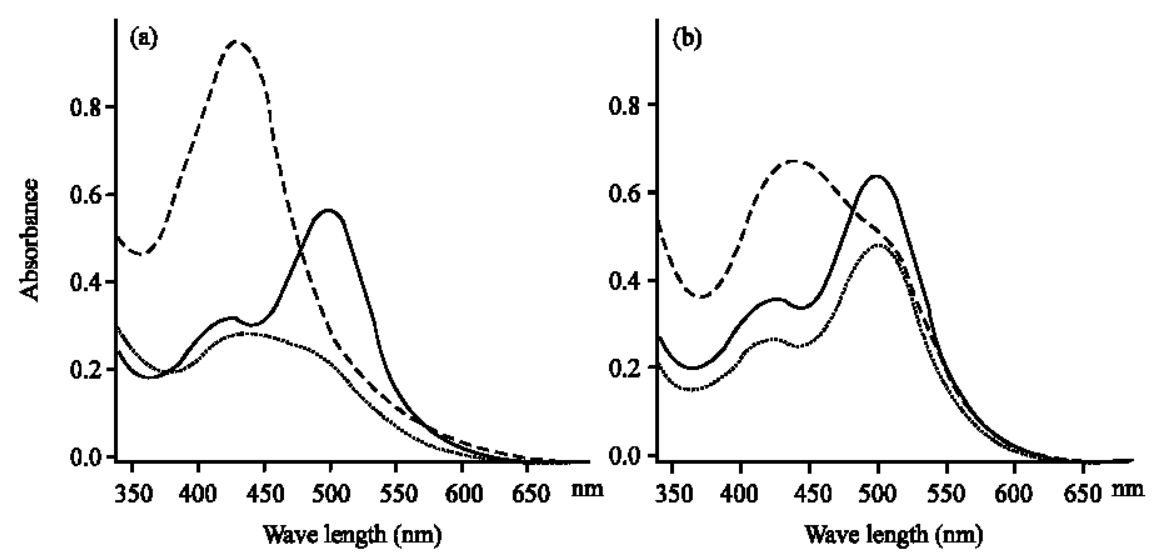

Fig. 12: Changes in the visible absorption spectra in solutions $C(a)$ and D (b) at days $0(-), 24(-\cdots)$ and $80(\ldots)$ of the assay

the disappearance of the anthocyanin and the progressive accumulation of xanthylium pigments. The colour changes observed were similar in the three solutions containing flavanols, although they were faster in solutions $\mathrm{C}$ and $\mathrm{E}$ than in solution $\mathrm{D}$, in agreement with its slower decrease of the anthocyanin and accumulation of xanthylium pigments due to the restriction of oxygen.

\section{CONCLUSION}

In this study further contributions are made to the understanding of the mechanisms involved in anthocyanin instability in food. The disappearance of Pg 3 gluc, by far the most important anthocyanin in strawberry, was studied in the absence and presence of flavanols and/or oxygen in aqueous acidic media. As it could be expected, the decrease of the anthocyanin was much slower when oxygen was avoided; however, the presence of flavanols constituted a destabilizing factor. Indeed, it was found that the effect of the flavanols even counteracted the protection offered by the anaerobic storage. In the solutions containing only Pg3gluc and oxygen, the anthocyanin breakdown to colourless products constituted the most important pathway for disappearance. However, in anaerobic conditions or in the presence of flavanols that mechanism does not represent a relevant way for anthocyanin loss. This, in the presence of flavanols, condensation with the anthocyanin yielding both colourless products and new xanthylium pigments was found to be the most important mechanism for anthocyanin disappearance. Conclusions about the identity of the products formed in all these reactions were obtained based on their mass spectra and $\mathrm{MS}^{2}$ fragmentation patterns and the structure of some of the newly-formed compounds was tentatively established for the first time. The results obtained are expected to contribute not only to interpret the processes of anthocyanin disappearance but they might also provide the basis to define the conditions for a better preservation of the red colour in strawberry-derived products.

\section{ACKNOWLEDGMENTS}

Financial support of Junta de Castilla y Leon (Spain) through the grant ref. SA086/01 is greatly acknowledged. Author Lopes da Silva was funded by European Union and Government of Portugal through the Programme PRODEP III (2001-2003). 


\section{REFERENCES}

Bakker, J., A. Piccineli and P. Bridle, 1993. Model wine solutions: Colour and composition changes during ageing. Vitis, 32: 111-118.

Bakker, J. and C.F. Timberlake, 1997. Isolation, identification and characterization of new color-stable anthocyanins occurring in some red wines. J. Agric. Food Chem., 45: 35-43.

Bakker, J., P. Bridle, T. Honda, H. Kuwano and N. Saito et al., 1997. Isolation and identification of a new anthocyanin occurring in some red wines. Phytochemistry, 44: 1375-1382.

Baublis, A., A. Spomer and M.D. Berber-Jimenez, 1994. Anthocyanin pigments: Comparison of extract stability. J. Food Sci., 59: 1219-1221.

Benabdeljalil, C., V. Cheynier, H. Fulcrand, A. Hakiki, M. Mosaddak and M. Moutounet, 2000. Identification of new pigments formed by reaction between anthocyanins and yeast metabolites (In French). Sci. Aliments, 20: 203-220.

Bishop, P.D. and C.W. Nagel, 1984. Characterization of the condensation product of malvidin 3, 5-diglucoside and catechin. J. Agric. Food Chem., 32: 1022-1026.

Boulton, R., 2001. The copigmentation of anthocyanins and its role in the color of red wine: A critical review. Am. J. Enol. Vitic., 52: 67-87.

Cameira dos Santos, P.J., J.M. Brillouet, V. Cheynier and M. Moutounet, 1996. Detection and partial characterisation of new anthocyanin-derived pigments in wine. J. Sci. Food Agric., 70: 204-208.

Cemeroglu, B., S. Velioglu and S. Isik, 1994. Degradation kinetics of anthocyanins in sour cherry juice and concentrate. J. Food Sci., 59: 1216-1218.

Clydesdale, F.M., J.H. Main, F.J. Francis and J.R.R.A. Damon, 1978. Concord grape pigments as colorants for beverages and gelatin desserts. J. Food Sci., 43: 1687-1692.

de Freitas, V., 1995. Studies on condensed tannins: Structures and proprieties of grape and wine procyanidins (In French), Ph.D. Thesis, Université de Bordeaux II, France.

de Freitas, V., Y. Glories and N. Laguerre, 1998. Incidence of molecular structure in oxidation of grape seeds procyanidins. J. Agric. Food Chem., 46: 376-382.

de Pascual-Teresa, S., Y. Gutierrez-Fernandez, J.C. Rivas-Gonzalo and C. Santos-Buelga, 1998. Characterization of monomeric and oligomeric flavan-3-ols from unripe almond fruit. Phytochem. Anal., 9: 21-27.

de Pascual-Teresa, S., J.C. Rivas-Gonzalo and C. Santos-Buelga, 2000a. Prodelphinidins and related flavanols in wine. Int. J. Food Sci. Technol., 35: 33-40.

de Pascual-Teresa, S., C. Santos-Buelga and J.C. Rivas-Gonzalo, 2000b. Quantitative analysis of flavan-3-ols in Spanish foodstuffs and beverages. J. Agric. Food Chem., 48: 5331-5337.

Dueñas, M., H. Fulcrand and V. Cheynier, 2006. Formation of anthocyanin-flavanol adducts in model solutions. Anal. Chim. Acta, 563: 15-25.

Escribano-Bailón, M.T., C. Santos-Buelga, G.L. Alonso and M.R. Salinas, 2002. Anthocyanin composition of Coriaria myrtifolia L. fruit. Phytochem. Anal., 13: 354-357.

Es-Safi, N.E., C. Le Guernevé, V. Cheynier and M. Moutounet, 1999. New polyphenolic compounds with xanthylium skeletons formed through reaction between $(+)$-catechin and glyoxylic acid. J. Agric. Food Chem., 47: 5211-5217.

Francia-Aricha, E.M., M.T. Guerra, J.C. Rivas-Gonzalo and C. Santos-Buelga, 1997. New anthocyanin pigments formed after condensation with flavanols. J. Agric. Food Chem., 45: 2262-2266.

Francia-Aricha, E.M., J.C. Rivas-Gonzalo and C. Santos-Buelga, 1998. Effect of malvidin-3monoglucoside on the browning of monomeric and dimeric flavanols. Eur. Food Res. Technol., 209: 223-228.

Friedrich, W., A. Eberhardt and R. Galensa, 2000. Investigation of proanthocyanidins by HPLC with electrospray ionization mass spectrometry. Eur. Food Res. Technol., 211: 56-64. 
Fulcrand, H., P.J. Cameira-dos-Santos, P. Sarni-Manchado, V. Cheynier and J. Fabre-Bonvin, 1996. Structure of new anthocyanin-derived wine pigments. J. Chem. Soc. Perkin Trans., 1: 735-739.

Fulcrand, H., V. Cheynier, J. Oszmianski and M. Moutounet, 1997. An oxidized tartaric acid residue as a new bridge potentially competing with acetaldehyde in flavan-3-ol condensation. Phytochemistry, 46: 223-227.

Fulcrand, H., C. Benabdeljalil, J. Rigaud, V. Cheynier and M. Moutounet, 1998. A new class of wine pigments generated by reaction between pyruvic acid and grape anthocyanins. Phytochemistry, 47: 1401-1407.

Furtado, P., P. Figueiredo, H. Chaves-das-Neves and F. Pina, 1993. Photochemical and thermal degradation of anthocyanidins. J. Photochem. Photobiol. A-Chem., 75: 113-118.

Garzon, G.A. and R.E. Wrolstad, 2001. The stability of pelargonidin-based anthocyanins at varying water activity. Food Chem., 75: 185-196.

Garzon, G.A. and R.E. Wrolstad, 2002. Comparison of the stability of pelargonidin-based anthocyanins in strawberry juice and concentrate. J. Food Sci., 67: 1288-1299.

Giusti, M.M. and R.E. Wrolstad, 1996. Radish anthocyanin extract as a natural red colorant for maraschino cherries. J. Food Sci., 61: 688-694.

Goiffon, J.P., P.P. Mouly and E.M. Gaydou, 1999. Anthocyanic pigment determination in red fruit juices, concentrated juices and syrups using liquid chromatography. Anal. Chim. Acta, 382: $39-50$.

Gonzalez-Paramas, A.M., F. Lopes da Silva, P. Martin-Lopez, G. Macz-Pop, S. Gonzalez-Manzano, C. Alcalde-Eon and J.J. Perez-Alonso et al., 2006. Flavanol anthocyanin condensed pigments in plant extracts. Food Chem., 94: 428-436.

Hayasaka, Y. and R.E. Asenstorfer, 2002. Screening for potential pigments derived from anthocyanins in red wine using nanoelectrospray tandem mass spectrometry. J. Agric. Food Chem., 50: 756-761.

Hermosín Gutiérrez, I., E. Sánchez-Palomo Lorenzo and A. Vicario Espinosa, 2005. Phenolic composition and magnitude of copigmentation in young and shortly aged red wines made from the cultivars, Cabernet Sauvignon, Cencibel and Syrah. Food Chem., 92: 269-283.

Hrazdina, G. and A.J. Franzese, 1974. Oxidation products of acylated anthocyanins under acidic and neutral conditions. Phytochemistry, 13: 231-234.

Jurd, L. and A.C. Waiss, 1965. Anthocyanins and related compounds-VI: Flavylium saltphloroglucinol condensation products. Tetrahedron, 21: 1471-1483.

Jurd, L., 1967. Catechin flavylium salt condensation reactions. Tetrahedron, 23: 1057-1064.

Jurd, L., 1969. Review of polyphenol condensation reactions and their possible occurrence in the aging of wines. Am. J. Enol. Vitic., 20: 191-195.

Kallio, H., M. Hakala, A.M. Pelkkikangas and A. Lapveteläinen, 2000. Sugars and acids of strawberry varieties. Eur. Food Res. Technol., 212: 81-85.

Kunsági-Máté, S., K. Szabó, M. Nikfardjam and L. Kollár, 2006. Determination of the thermodynamic parameters of the complex formation between malvidin-3-O-glucoside and polyphenols. Copigmentation effect in red wines. J. Biochem. Biophys. Meth., 69: 113-119.

Liao, H., Y. Cai and E. Haslam, 1992. Polyphenol interactions, Part 6, Anthocyanins: Copigmentation and colour changes in red wines. J. Sci. Food Agric., 59: 299-305.

Lopes da Silva, F., S. de Pascual-Teresa, J.C. Rivas-Gonzalo and C. Santos-Buelga, 2002. Identification of anthocyanin pigments in strawberry (cv Camarosa) by LC using DAD and ESI-MS detection. Eur. Food Res. Technol., 214: 248-253.

Lopes da Silva, F., M.T. Escribano-Bailon, J.J. Perez-Alonso, J.C. Rivas-Gonzalo and C. SantosBuelga, 2007. Anthocyanin pigments in strawberry. LWT-Food Sci. Technol., 40: 374-382.

Oszmianski, J., J.C. Sapis and J.J. Macheix, 1985. Changes in grape seed phenols as affected by enzymic and chemical oxidation in vitro. J. Food Sci., 50: 1505-1506. 
Özkan, M., 2002. Degradation of anthocyanins in sour cherry and pomegranate juices by hydrogen peroxide in the presence of added ascorbic acid. Food Chem., 78: 499-504.

Özkan, M., A. Yemenicioglu, N. Asefi and B. Cemeroglu, 2002. Degradation kinetics of anthocyanins from sour cherry, pomegranate and strawberry juices by hydrogen peroxide. J. Food Sci., 67: $525-529$.

Piffaut, B., F. Kader, M. Girardin and M. Metche, 1994. Comparative degradation pathways of malvidin 3,5-diglucoside after enzymatic and thermal treatments. Food Chem., 50: 115-120.

Poei-Langston, M.S. and R.E. Wrolstad, 1981. Color degradation in an ascorbic-acid anthocyaninflavanol model system. J. Food Sci., 46: 1218-1222.

Remy-Tanneau, S., C. Le Guernevé, E. Meudec and V. Cheynier, 2003. Characterization of a colorless anthocyanin-flavan-3-ol dimer containing both carbon-carbon and ether interflavanoid linkages by NMR and mass spectrometry. J. Agric. Food Chem., 51: 3592-3597.

Rivas-Gonzalo, J.C., S. Bravo-Haro and C. Santos-Buelga, 1995. Detection of compounds formed through the reaction of malvidin 3-monoglucoside and catechin in the presence of acetaldehyde, J. Agric. Food Chem., 43: 1444-1449.

Romero, C. and J. Bakker, 1999. Interactions between grape anthocyanins and pyruvic acid, with effect of $\mathrm{pH}$ and acid concentration on anthocyanin composition and color in model solutions. J. Agric. Food Chem., 47: 3130-3139.

Salas, E., H. Fulcrand, E. Meudec and V. Cheynier, 2003. Reactions of anthocyanins and tannins in model solutions. J. Agric. Food Chem., 51: 7951-7961.

Salas, E., M. Dueñas, M. Schwarz, P. Winterhalter, V. Cheynier and H. Fulcrand, 2005. Characterization of pigments from different High Speed Countercurrent Chromatography wine fractions. J. Agric. Food Chem., 53: 4536-4546.

Santos-Buelga, C., S. Bravo-Haro and J.C. Rivas-Gonzalo, 1995. Interactions between catechin and malvidin-3-monoglucoside in model solutions. Z Lebensm Unters Forsch, 201: 269-274.

Santos-Buelga, C., E.M. Francia-Aricha, S. de Pascual-Teresa and J.C. Rivas-Gonzalo, 1999. Contribution to the identification of the pigments responsible for the browning of anthocyaninflavanol solutions. Eur. Food Res. Technol., 209: 411-415.

Santos-Buelga, C., C. Garcia-Viguera and F.A. Tomas-Barberan, 2003. On-line identification of flavonoids by HPLC coupled to diode array detection. In: Methods in Polyphenol Analysis. Royal Society of Chemistry (Cambridge, UK), pp: 92-127.

Sarni-Manchado, P., V. Cheynier and M. Moutounet, 1997. Reactions of polyphenoloxidase generated caftaric acid o-quinone with malvidin 3-glucoside. Phytochemistry, 45: 1367-1369.

Somers, T.C., 1971. The polymeric nature of wine pigments. Phytochemistry, 10: 2175.

Somers, T.C. and M.E. Evans, 1986. Evolution of red wines, I, Ambient influences on colour composition during early maturation. Vitis, 25: 31-39.

Thompson, R.S., D. Jacques, E. Haslam and R.J.N. Tanner, 1972. Plant proanthocyanidins, Part I, introduction; the isolation, structure and distribution in nature of plant procyanidins. J. Chem. Soc. Perkin Trans., 1: 1387-1399.

Timberlake, C.F. and P. Bridle, 1976. Interactions between anthocyanins, phenolic compounds and acetaldehyde and their significance in red wines. Am. J. Enol. Vitic., 27: 97-105.

Vidal, S., E. Meudec, V. Cheynier, G. Skouroumounis and Y. Hayasaka, 2004. Mass spectrometric evidence for the existence of oligomeric anthocyanins in grape. J. Agric. Food Chem., 521: 7144-7151. 